Supporting Information online for:

\title{
Site Partition: Turning One Site into Two for Adsorbing $\mathrm{CO}_{2}$
}

\author{
Ziqi Tian, ${ }^{1}$ Sheng Dai, ${ }^{2,3}$ De-en Jiang ${ }^{1, *}$ \\ ${ }^{1}$ Department of Chemistry, University of California, Riverside, California 92521, United States \\ ${ }^{2}$ Chemical Sciences Division, Oak Ridge National Laboratory, Oak Ridge, Tennessee 37831-6201, \\ United States \\ ${ }^{3}$ Department of Chemistry, The University of Tennessee, Knoxville, Tennessee 37996-1600, United \\ States \\ *E-mail: de-en.jiang@ucr.edu. Telephone: +1-951-827-4430
}

Computational details: Based on experimental crystal structures of CPM-33 family MOFs ${ }^{1}$ and TDCOF- $5,{ }^{2}$ we performed DFT calculation with Vienna ab initio simulation package (VASP) ${ }^{3}$, to relax geometries, obtain $\mathrm{CO}_{2}$ binding energies, and generate the electrostatic potential. The generalized gradient approximation in the form of Perdew-Burke-Ernzerhof functional (GGA$\mathrm{PBE})^{4}$ was employed to describe electron exchange correlation. We used the projector augmented wave (PAW) ${ }^{5}$ pseudopotential with energy cutoff at $400 \mathrm{eV}$ for the planewaves. Brillouin zone sampling was done for the $\Gamma$-point only. To accurately describe van der Waals interaction, dispersion correction termed DFT-D3 was employed. ${ }^{6}$ Spin-polarization was included to account for the spin states of the $\left[\mathrm{Ni}_{3} \mathrm{OH}\right]^{6+}$ cluster in CPM-33 MOFs. From the DFT-based electrostatic potential, atomic partial charges were determined by the REPEAT method. ${ }^{7}$ Then we carried out grand canonical Monte Carlo (GCMC) simulation with the MUSIC code ${ }^{8}$ to reproduce $\mathrm{CO}_{2}$ absorption isotherms at $298 \mathrm{~K}$ (Figure S1) and $273 \mathrm{~K}$ (Figure S2) for CPM-33 MOFs. The interactions of gas-sorbent and gas-gas were modelled as the sum of electrostatic and Lennard-Jones (LJ) 12-6 non-bonded potentials, based on REPEAT-derived atomic charges and the Dreiding force field, ${ }^{9}$ respectively. $\mathrm{CO}_{2}$ was described by a three-site model, in which carbon and oxygen possess charges of $0.576|\mathrm{e}|$ and $-0.278|\mathrm{e}|,{ }^{10}$ respectively. 

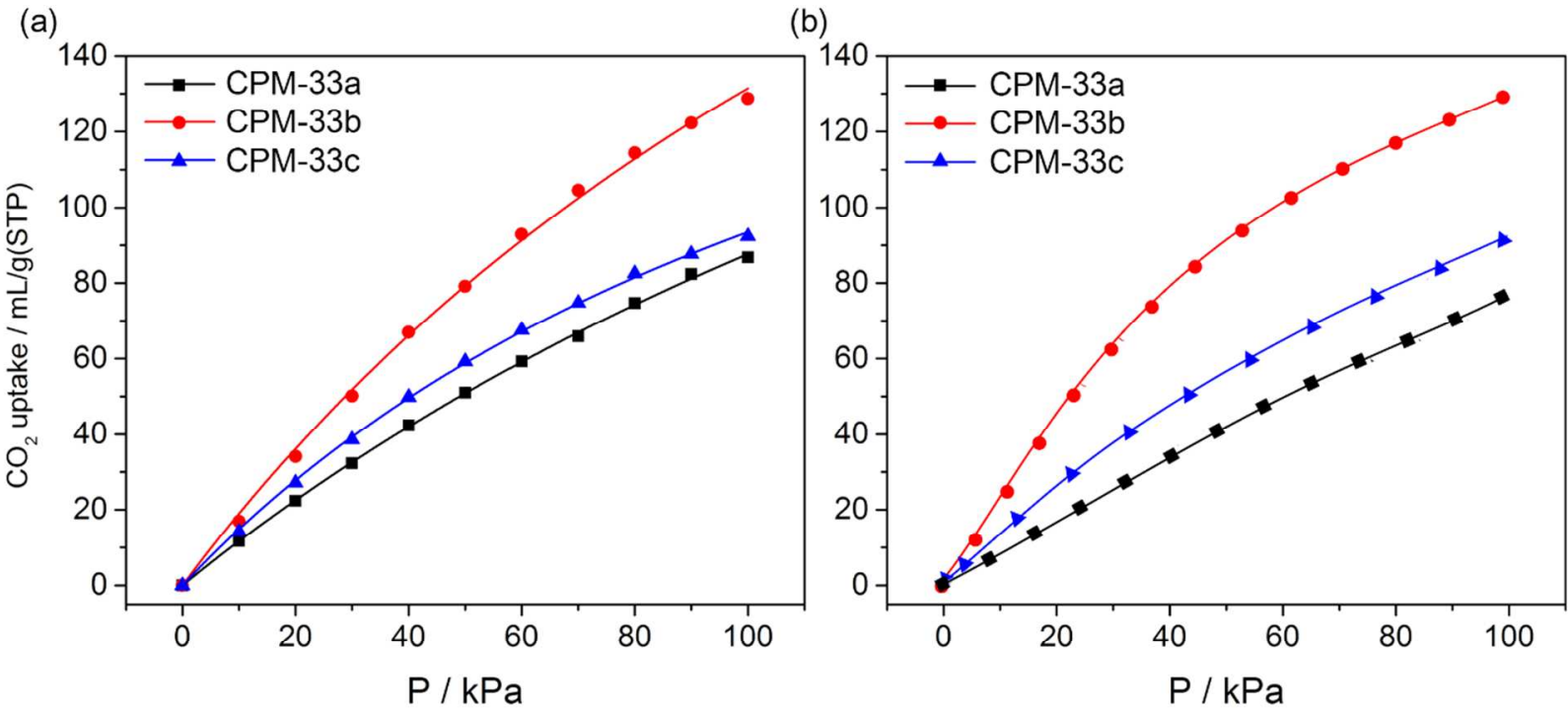

Figure S1. Comparison of (a) simulated and (b) experimental isotherms ${ }^{1}$ of CPM-33a, CPM-33b, and CPM-33c at $298 \mathrm{~K}$.
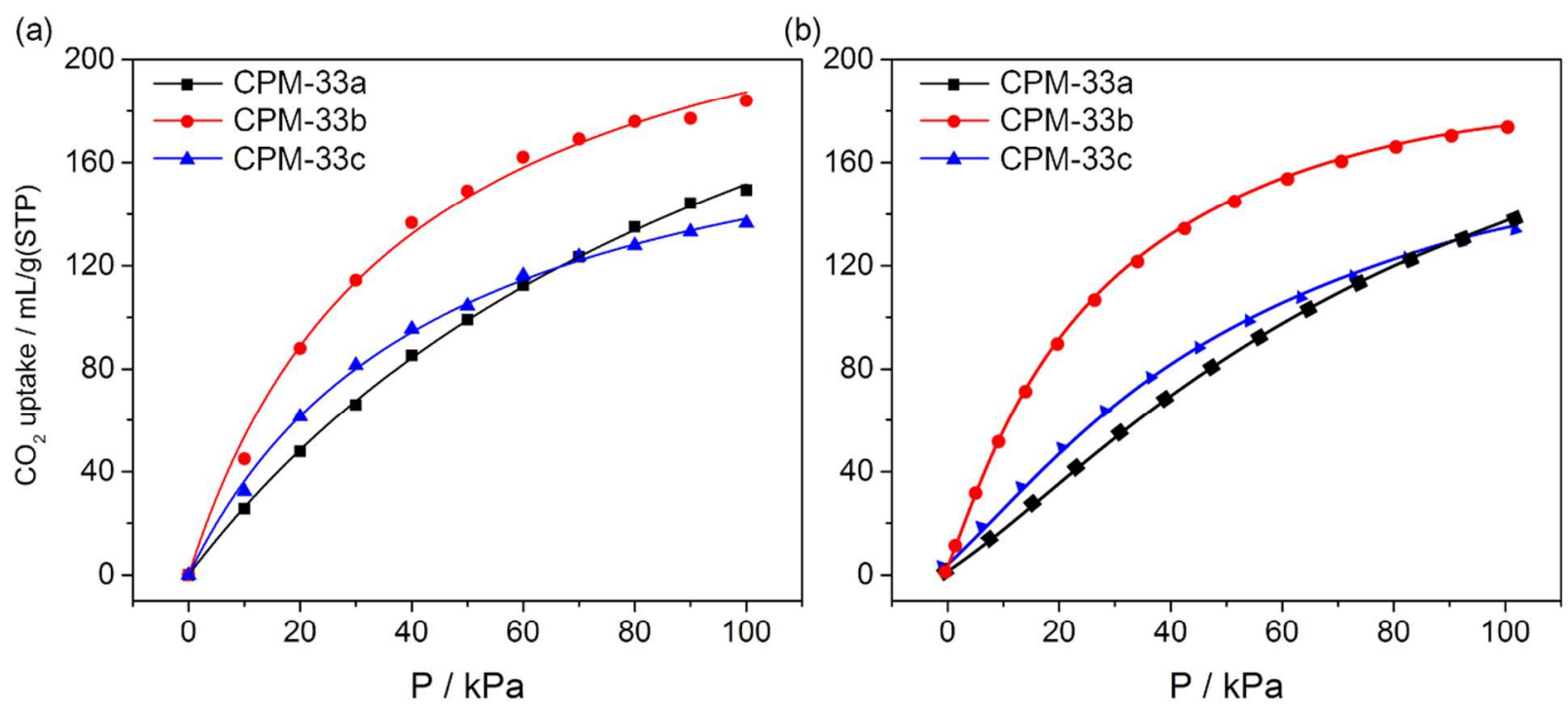

Figure S2. Comparison of (a) simulated and (b) experimental isotherms ${ }^{1}$ of CPM-33a, CPM-33b, and CPM-33c at $273 \mathrm{~K}$. 


\section{References}

(1) Zhao, X.; Bu, X. H.; Zhai, Q. G.; Tran, H.; Feng, P. Y. Pore Space Partition by Symmetry-

Matching Regulated Ligand Insertion and Dramatic Tuning on Carbon Dioxide Uptake. J. Am. Chem. Soc. 2015, 137, 1396-1399.

(2) Kahveci, Z.; Islamoglu, T.; Shar, G. A.; Ding, R.; El-Kaderi, H. M. Targeted Synthesis of a Mesoporous Triptycene-Derived Covalent Organic Framework. CrystEngComm 2013, 15, 1524-1527.

(3) Kresse, G.; Furthmuller, J. Efficient Iterative Schemes for ab Initio Total-Energy Calculations Using a Plane-Wave Basis Set. Phys. Rev. B 1996, 54, 11169-11186.

(4) Perdew, J. P.; Burke, K.; Ernzerhof, M. Generalized Gradient Approximation Made Simple. Phys. Rev. Lett. 1996, 77, 3865-3868.

(5) Blochl, P. E. Projector Augmented-Wave Method. Phys. Rev. B 1994, 50, 17953-17979.

(6) Grimme, S.; Antony, J.; Ehrlich, S.; Krieg, H. A Consistent and Accurate ab Initio Parametrization of Density Functional Dispersion Correction (DFT-D) for the 94 Elements H-Pu. J. Chem. Phys. 2010, 132, 154104.

(7) Campana, C.; Mussard, B.; Woo, T. K. Electrostatic Potential Derived Atomic Charges for Periodic Systems Using a Modified Error Functional. J. Chem. Theory Comput. 2009, 5, 2866-2878.

(8) Gupta, A.; Chempath, S.; Sanborn, M. J.; Clark, L. A.; Snurr, R. Q. Object-Oriented Programming Paradigms for Molecular Modeling. Mol. Simul. 2003, 29, 29-46.

(9) Mayo, S. L.; Olafson, B. D.; Goddard, W. A. Dreiding - a Generic Force-Field for Molecular Simulations. J. Phys. Chem. 1990, 94, 8897-8909.

(10) Hirotani, A.; Mizukami, K.; Miura, R.; Takaba, H.; Miya, T.; Fahmi, A.; Stirling, A.; Kubo, M.; Miyamoto, A. Grand Canonical Monte Carlo Simulation of the Adsorption of $\mathrm{CO}_{2}$ on Silicalite and NaZSM-5. Appl. Surf. Sci. 1997, 120, 81-84. 